\title{
ESPMH News
}

\section{Forthcoming ESPMH Conferences}

Annual conference ESPMH, 2007

XXIth International Conference of the European Society for Philosophy of Medicine and Healthcare (together with the Cardiff Institute for Society, Health and Ethics). Theme: "Ethics, philosophy and public health". Date: August 15-18, 2007. Place: Cardiff, United Kingdom. Abstracts should be submitted before January 1, 2007. For more information: Bert Gordijn, PhD, Secretary of the ESPMH, Dept. of Ethics, Philosophy and History of Medicine, Radboud University Nijmegen Medical Centre, PO Box 9101, 6500 HB Nijmegen, The Netherlands. E-mail: b.gordijn@efg.umcn.nl.

\section{New Members}

Deczki, Gyöngyi, Kikerics 15, 1121 Budapest, Hongary

Heusser, Peter, Institute of Complementary Medicine, University Bern, KIKOM, Inselspital, Imhoof

Pavillon, CH-3010 Bern, Switzerland

Neubeck, Anna-Karin, Skinnarvikiringen 12, S-11727 Stockhokm, Sweden

Rhodes, Rosamond, 4621 Delafield Avenue, 10471 Bronx, NewYork, USA

Salmi, Anna, Tilanhoitajankaari 6 D84, 00790 Helsinki, Finland

Wingfield, Joy, 37 Blanford Gardens, West Bridgford, Nottingham NG2 7UQ, England, UK

\section{Cancellations}

Angelus, Tamas, Budapest, Hungary

Bullington, Jennifer, Stockholm, Sweden

Chapouthier, Georges, Paris, France

Dornberg, Martin, Freiburg, Germany

Grond-Ginsbach, Casper, Heidelberg, Germany

Hoedemaekers, Rogeer, Nijmegen, The Netherlands

Kolberg, Christiane, Tromsdalen, Noway

Körner, Uwe, Berlin, Germany

Nummenmaa, Juho, Tampere, Finland

Privetera, Salvatore, Acireale, Italy

Vandenbussche, Freddy, Middelkerke, Belgium 